\title{
Emotion Detecting System
}

\author{
Asst. Prof. Dr. Buthaina Fahran Abed ${ }^{1}$, Russul Haider Abed Ali ${ }^{2}$ \\ buthynna[at]yahoo.com, russellr605[at]gmail.com \\ 1Informatics Institute for Postgraduate Studies, UITC, Baghdad, Iraq \\ 2Research Scholar, Informatics Institute for Postgraduate Studies, UITC, Baghdad, Iraq
}

\begin{abstract}
Facial expression recognition has many potential applications that had attracted the attention of researchers in the last decade. In this paper, a proposed system for analyzing the facial emotions is presented. Where features are extracted by applying geometric of the distances that are determined by using the locations of the basic facial components through detecting mouth. Then evaluate them by using the standard dataset "CK" which have been used as test material for testing the results. The percentage of recognition was around $96 \%$ when the basic emotion classes were tested, which is considered to be high when compared with the results of other newly published works.
\end{abstract}

Keywords: Facial expression recognition, Automatic Processing, Facial Emotions, Facial components, Detecting Mouth

\section{Introduction}

Facial expression is a visible manifestation of the affective state, cognitive activity, intention, personality, and psychopathology of a person [1]. Since each person has different cultural and surrounding background, it is difficult to precisely determine the number of facial expressions that are used daily in our life. However, researchers on analyzing facial impressions have focused on seven basic expressive gestures which are: anger, disgust, fear, happiness, sadness, neutral, and surprise [2]. Till now, the recognition of facial expression still a problem with a big number of challenges. This is mainly due to the following reasons: (i) face detection from the captured image and segmentation process for the purpose of Region of Interest (ROI) extraction is a difficult task [3], (ii) Generating effective descriptors from ROI region (i.e., face region) that reduce the percentage difference between samples per class and increase between classes differences is an important and difficult step for building facial impression recognition system with high accuracy and (iii) difficulty of selection the classifier that classifies the face expression into correct emotional state [4][5][6].

Accurate extraction of facial components is dependent on preprocessing, that include (i) converted the input image from colored (RGB) to gray scale image equation 1 [7] [8] and (ii) noise removal of image, there is many type of noise removal as a Median filter (MED) size $(3 \times 3)$ as illustrated in equation 2 . Which is removal noise to blur the image (to reduce background texture effects)[9].

$$
\begin{aligned}
& \text { Gray Image }=0.299 \mathrm{R}+0.587 \mathrm{G}+0.114 \mathrm{~B} \text { eq. }(1) \\
& f_{3 \times 3}=\frac{1}{9}\left[\begin{array}{lll}
1 & 1 & 1 \\
1 & 1 & 1 \\
1 & 1 & 1
\end{array}\right]
\end{aligned}
$$

When preprocessing image needed detection and representing information of the image that is locate objects and boundaries (lines, curves, etc.) by image segmentation that are accomplished in (i) edge detection is fundamentally, a method of dividing an image into segments of discontinuity by locating the points, in a digital image, where there is an unexpected change in image intensities and. The most popular first order edge detection operators are Prewitt, Roberts, and Sobel which has been explained in [10].(ii) thresholding is a technique for image segmentation which can be considered as an old, popular and simple method. Although image segmentation by thresholding is a simple approach, but at the same time, it is a powerful approach for segmenting images especially when images have bright objects on dark background, or vice versa. Threshold technique value can be viewed global, local, and dynamic threshold which has been explained in [11].

$$
\begin{gathered}
T=T[\boldsymbol{x}, \boldsymbol{y}, \boldsymbol{p}(\boldsymbol{x}, \boldsymbol{y}), \boldsymbol{f}(\boldsymbol{x}, \boldsymbol{y})] \\
\boldsymbol{T}(\boldsymbol{x}, \boldsymbol{y})=\left\{\begin{array}{l}
\mathbf{0} \text { if } f(x, y) \leq p(x, y) \\
\mathbf{1} \text { otherwise }
\end{array}\right.
\end{gathered}
$$

Where:

$\mathrm{f}(\mathrm{x}, \mathrm{y})$ is the gray level of point $(\mathrm{x}, \mathrm{y})$, and $\mathrm{p}(\mathrm{x}, \mathrm{y})$ denotes some local property of this point such as the average gray level of a neighborhood centered on $(x, y)$.

If $\mathrm{T}$ depends on

1. $\mathrm{f}(\mathrm{x}, \mathrm{y})$ only - global threshold

2. Both $f(x, y) \& p(x, y)$ - local threshold

3. $(\mathrm{x}, \mathrm{y})$ - dynamic threshold

When determine the non-regular objects of the face using the (iii) region growing method which will eliminate the irrelevant background regions [12].

In geometric-based feature extraction methods; which are commonly adopted in many research areas like face recognition and facial emotion recognition. In these methods the location of key facial components (such as mouth, eyes, eyebrows and nose) are being tracked and the geometric relationship between certain key points (fiducial points) on the face (as distances, angles and shapes) are determined when making cognition decision. The established feature vectors for performing facial cognition tasks usually transmit the extracted facial components at these key geometric regions on face [13]. In general, there are two main methods for facial emotion feature generation: geometric- based features and appearancebased features. Geometric features always present in the

\section{Volume 6 Issue 12, December 2017}




\section{International Journal of Science and Research (IJSR) \\ ISSN (Online): 2319-7064 \\ Index Copernicus Value (2016): 79.57 | Impact Factor (2015): 6.391}

face but may be deformed due to any kind of facial expression. One of this operations calculating distances (D) between any two points $(\mathrm{P}, \mathrm{Q})$ is calculated by using the Euclidean distance metric as follows in equation 5

$$
d_{x, y}=\sqrt[0]{\left(x_{2}-x_{1}\right)^{2}+\left(y_{2}-y_{1}\right)^{2}}
$$

When calculated the length of $\mathrm{x}$ and $\mathrm{y}$ which are the following equations:

$$
\begin{aligned}
& |\mathbf{x}|=\left|\mathbf{x}_{2}-\mathbf{x}_{1}\right| \\
& |\mathbf{y}|=\left|\mathbf{y}_{2}-\mathbf{y}_{1}\right|
\end{aligned}
$$$$
\text { eq.(6) }
$$

The basic components of the face or the points that represent facial features are extracted from the image spatial domain for purpose of classification can be used to get discriminating geometrical based features [14].

The paper is organized as follows. Section II describes the adopted methodology. Section III explains the results obtained, followed by conclusion and future work in Section IV. In this project first start the preprocessing on the color image input. Next, identify the mouth of the person and classify the whether the person happy, angry, and surprise. The goal is to extract the mouth which is the basic facial component for images containing one face in front position regardless of image illumination.

\section{The Proposed System}

The proposed system block diagram which is illustrated in figure 1 consists of four main stages.

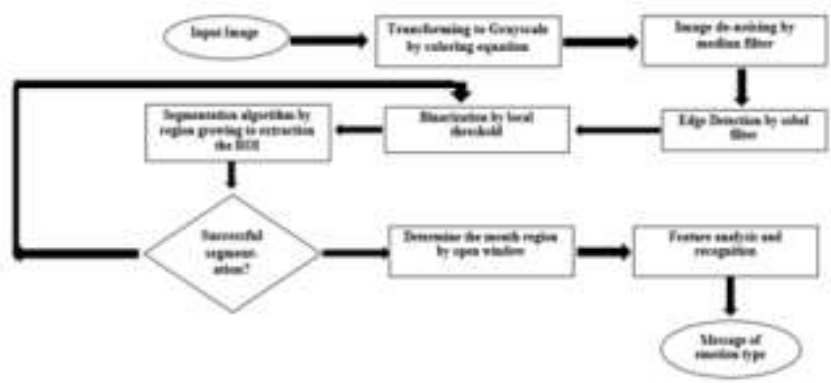

Figure 1: The proposed system block diagram

\subsection{Preprocessing stage}

In this stage the image is cleared. The system starts with the preprocessing module which consists of two steps:

(i) Transforming the input image to grayscale step: which converts the input image into gray image by using equation (1) true color (RGB) in algorithm 1.

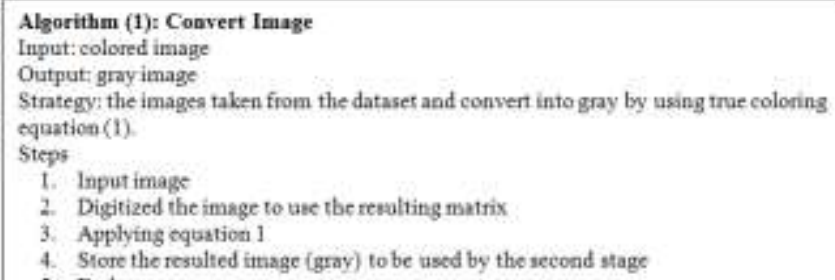

(ii) Image de-noising step: Which remove the noise from the gray image that was produced from previous step as illustrate algorithm 2 .

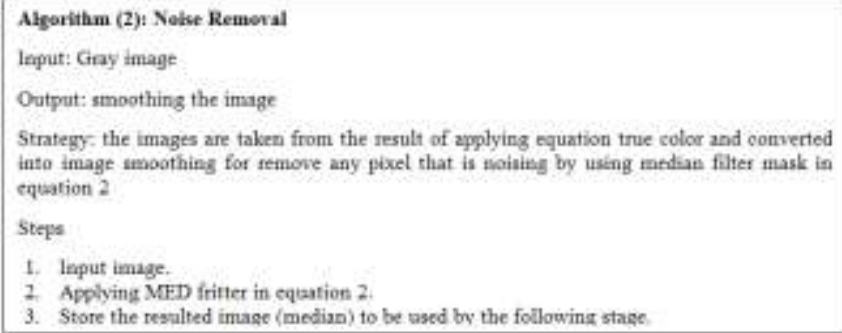

\subsection{Segmentation stage}

The segmentation module which consist of three steps:

Step1. Edge detection step: is applied on the cleared image that was produced from the preprocessing stage.In this step the edge image is extracted using the sobel edge detector and the edges at those points where the gradient of an image is maximum. In this step uses two filter size 3 x 3 kernels. One for changes in the horizontal direction, and one for changes in the vertical direction as shown in equation 8 and 9 [15].

$$
\begin{aligned}
& G_{x}=\left[\begin{array}{ccc}
-1 & 0 & +1 \\
-2 & 0 & +2 \\
-1 & 0 & +1
\end{array}\right] \\
& G_{y}=\left[\begin{array}{ccc}
+1 & +2 & +1 \\
0 & 0 & 0 \\
-1 & -2 & -1
\end{array}\right]
\end{aligned}
$$

Typically, an approximate magnitude is computed by using equation 10 :

$$
|\boldsymbol{G}|=|\boldsymbol{G} \boldsymbol{x}|+|\boldsymbol{G} \boldsymbol{y}|
$$

Where: $G x$ is a horizontal sobel mask and vertical sobel mask $G y$.

Step2. Thresholding: is applied on the image which was detected in the previous step where converting to the binary. Then determine the value of the threshold to isolate the object from the background. After that the value of the threshold can be applying on local threshold operation $\mathrm{T}(x, y)$ on the edge of the image using equation 4.

Step3. Segmentation algorithm step: is applied on the image which was detected in the previous step. Which was performed through algorithm 3 . 


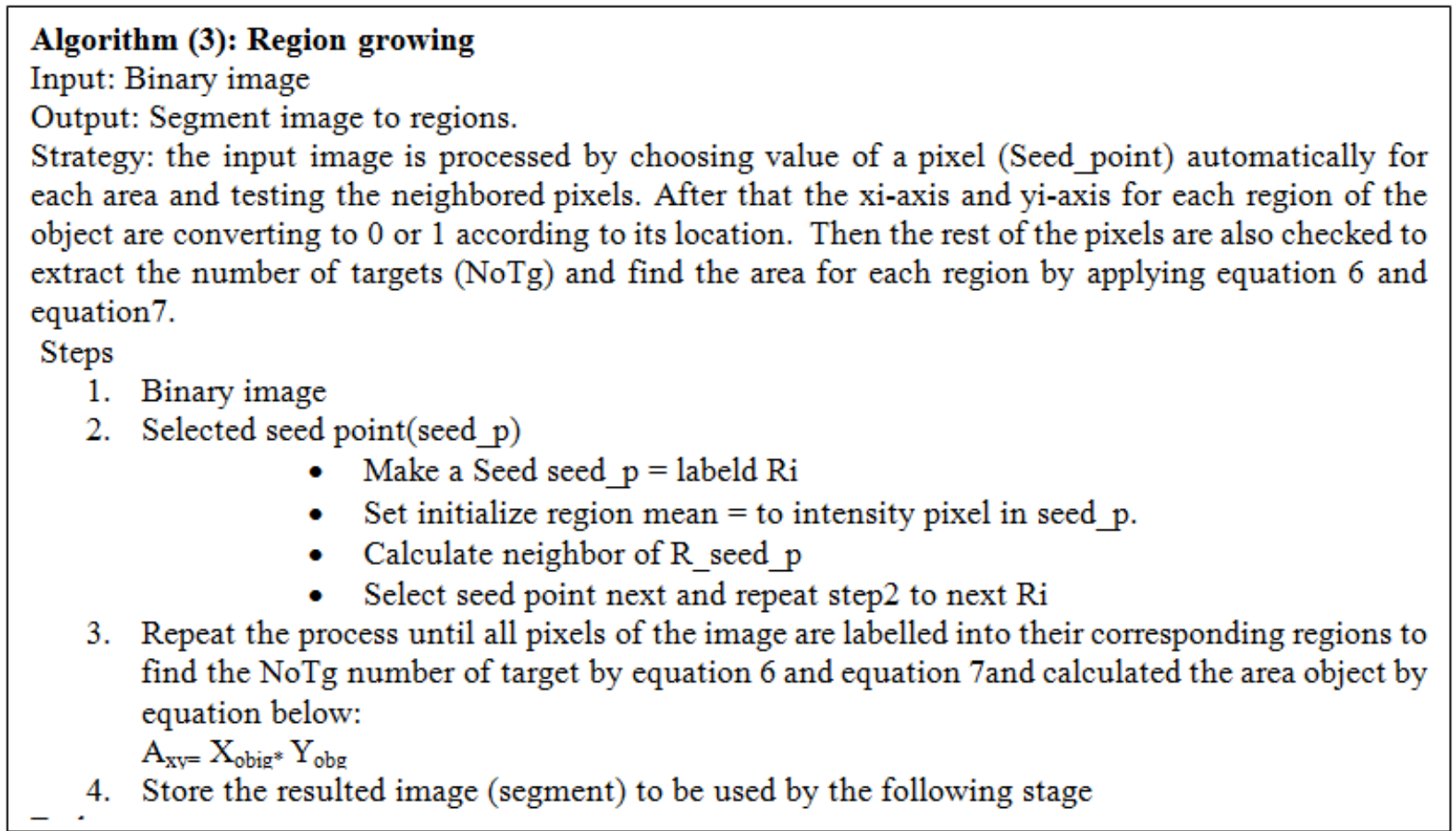

\subsection{Regions of interest (ROI) extraction stage}

This stage allocates and determine the mouth region by using algorithm 4.ROIs) that are necessary which will be used to determine the emotion type in the system as shown in the mouth detection algorithm 4 . A window is created to allocate mouth location in the image which is based on the nature human facial morphology as below: a. The face always contains one mouth and should be in the bottom of the face and must be on the same baseline level of symmetric line.

b. The segment must be under nose point, but not far from it.

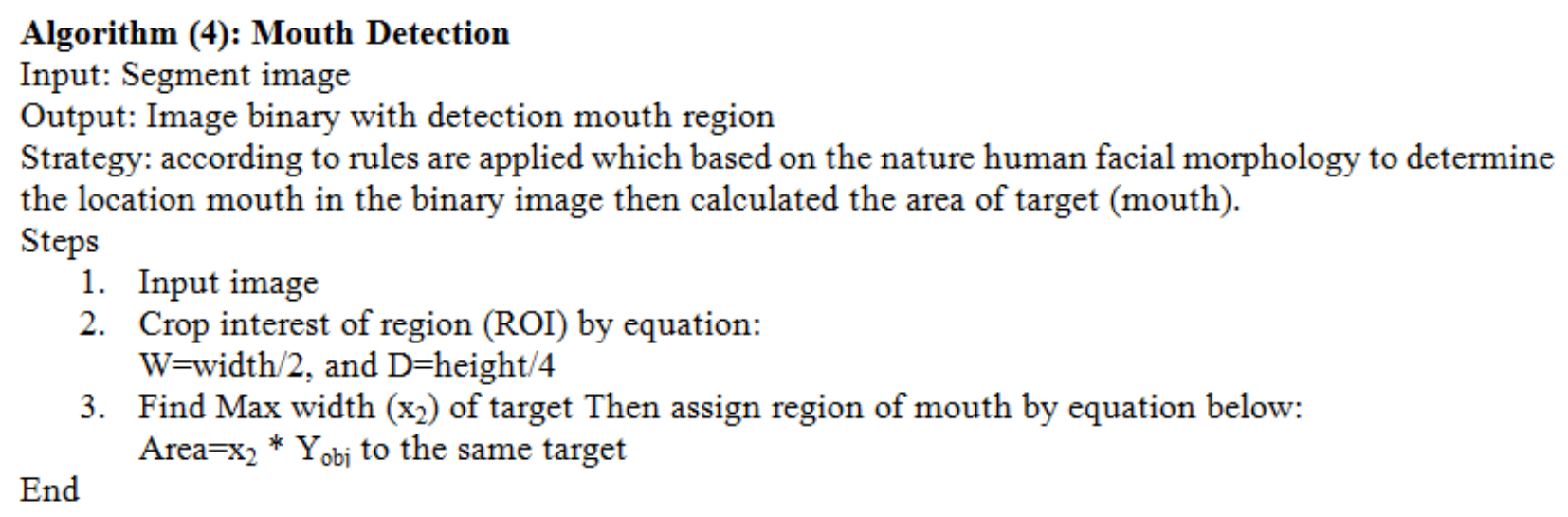

2. Crop interest of region (ROI) by equation: $\mathrm{W}=$ width $/ 2$, and $\mathrm{D}=$ height $/ 4$

3. Find Max width $\left(\mathrm{x}_{2}\right)$ of target Then assign region of mouth by equation below: Area $=\mathrm{x}_{2} * \mathrm{Y}_{\text {obi }}$ to the same target

End

\subsection{Feature extraction stage}

In this stage geometric operations calculating the distances between two coordinating in the mouth region by using equation 5. The feature extraction module is applied after extracting the interested regions from the image produced from the previous stage.
Then feature analysis and recognition is applied geometrybased methods to feature analysis of the mouth region after extraction will be recognition features by a message entitled the nature of the emotion (Angry, Happy, and surprise) and illustrated in recognition emotions algorithm (5). 


\section{International Journal of Science and Research (IJSR) \\ ISSN (Online): 2319-7064}

Index Copernicus Value (2016): 79.57 | Impact Factor (2015): 6.391

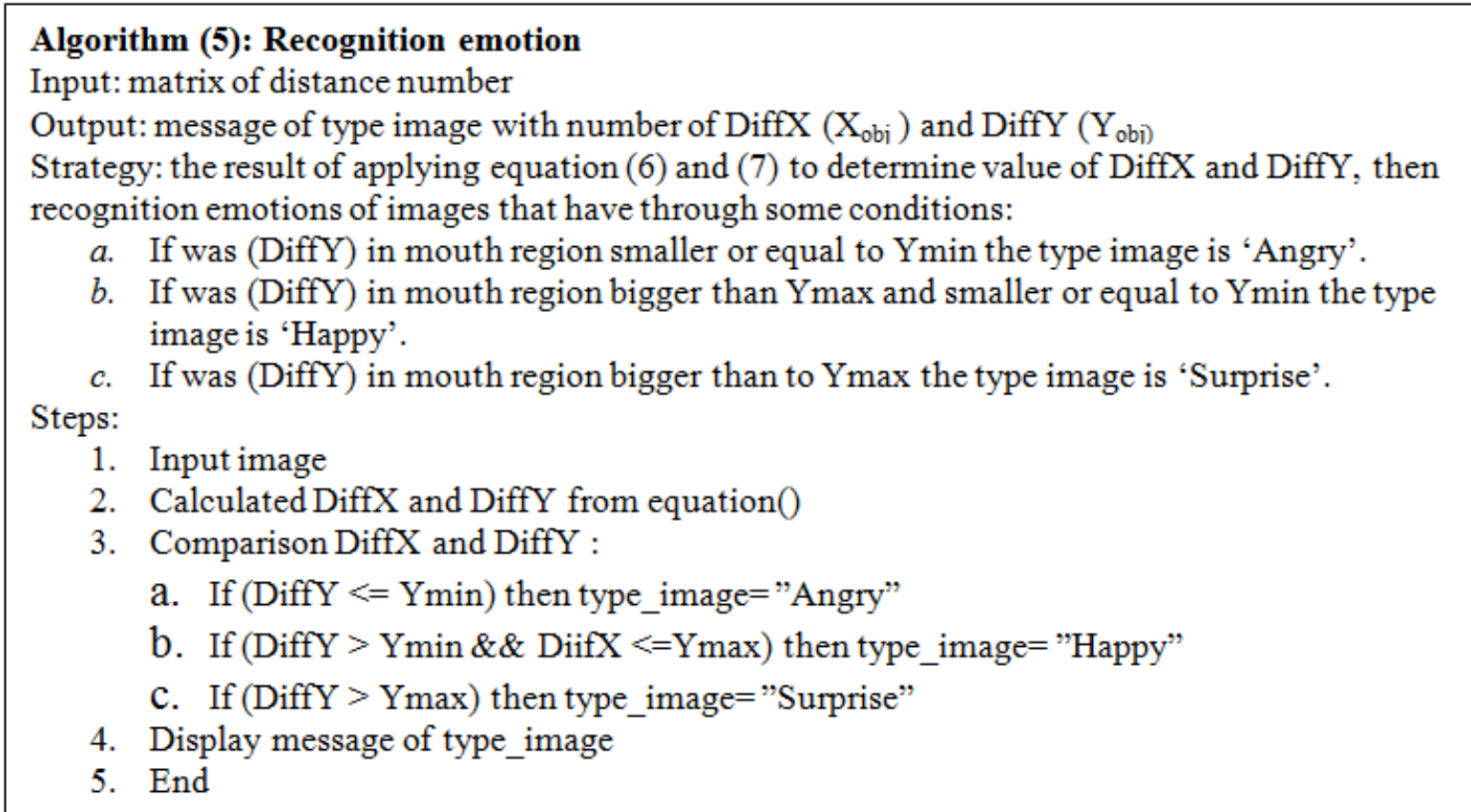

\subsection{Performance Parameters}

The recognition rate (RR) measure[16] in equation 6 , was used for studying the performance of the propoced system. The number of images image that was used for testing are 222 images.

$R R_{E}=\frac{\text { Number of the image crrectily recognition }}{\text { Total number of the image test }} \times 100$ eq.(11)

\subsubsection{Emotions Recognition Rate}

This emotion is shown in figures 2 that are correctly which recognition them $96 \%$ while not recognition is $4 \%$.

Where, the number of the emotion image that recognition is 214 images of the total 222 images, whereas the images are not recognizing their number was 8 images. The figure 2 illustrates RR for each emotion (angry, surprise, and happy).

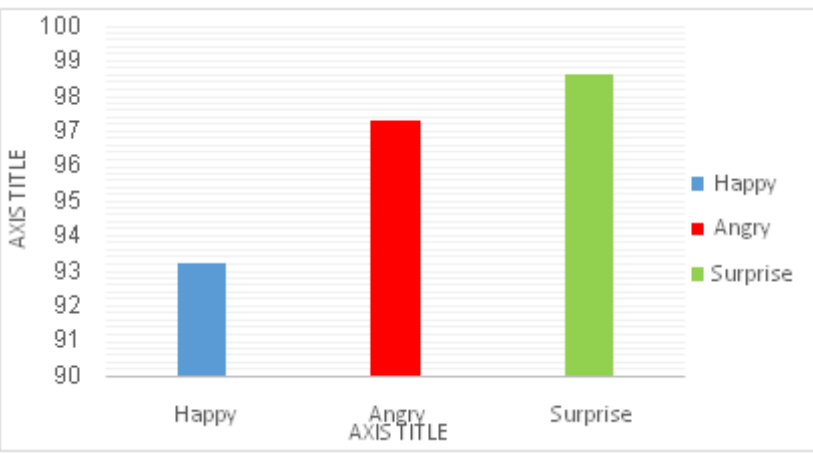

Figure 2: Recognition emotion rate

\section{A. The Surprise Emotions}

This emotion is shown in figure 3 the result of applying equation 11 that are correctly which recognition them $99 \%$ while not recognition is $1 \%$.

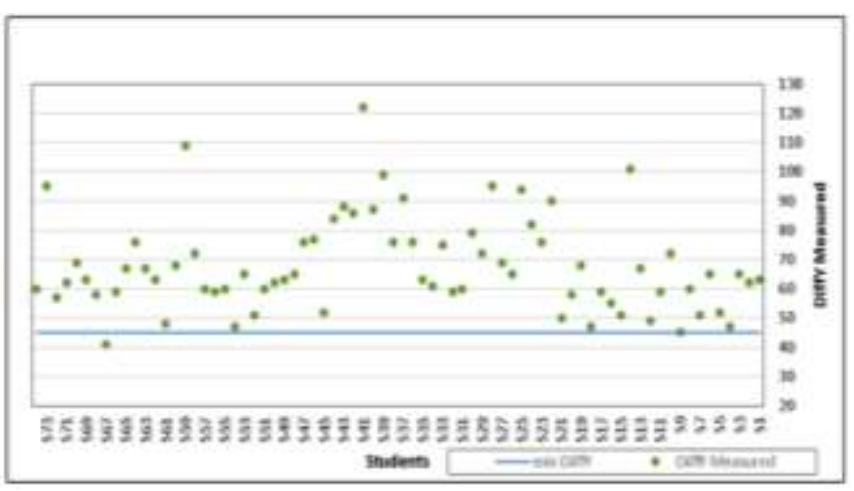

Figure 3: Surprise Emotion Image

\section{B. The Angry Emotions}

This emotion is shown in figure 4 that are correctly which recognition them $97 \%$ while not recognition is $3 \%$.

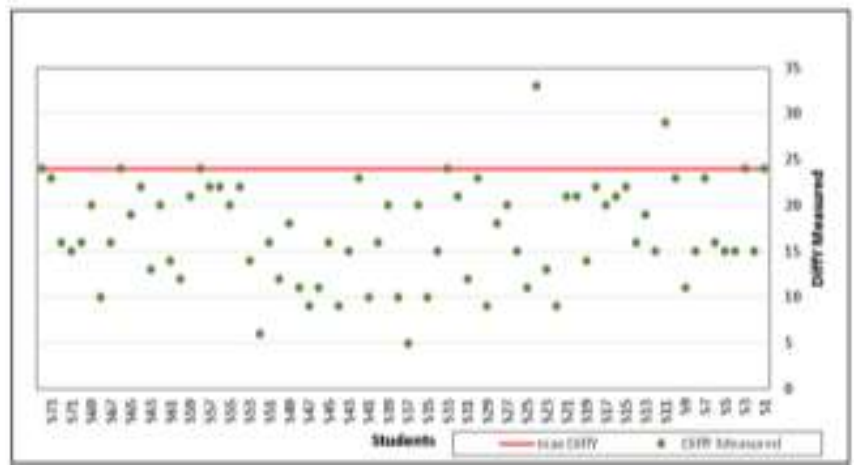

Figure 4: The angry emotion for person

\section{The Happy Emotions}

This emotion is shown in figure 5 that is correctly which recognition them $93 \%$ while not recognition is $7 \%$.

\section{Volume 6 Issue 12, December 2017}




\section{International Journal of Science and Research (IJSR) \\ ISSN (Online): 2319-7064}

Index Copernicus Value (2016): 79.57 | Impact Factor (2015): 6.391

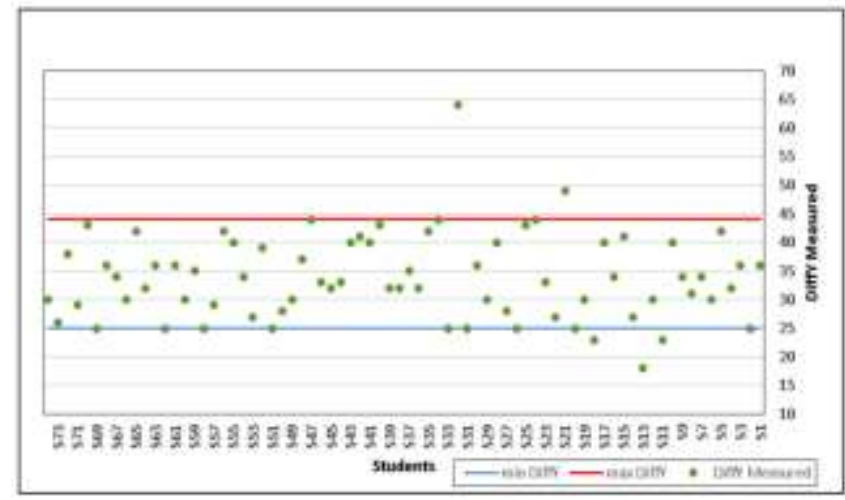

Figure 5: The happy emotion Image

Table 1: The comparison of recognition rate with some other people's independed systems

\begin{tabular}{|c|c|c|}
\hline Authors & $\begin{array}{c}\text { Feature Extraction } \\
\text { Method }\end{array}$ & RR \\
\hline $\begin{array}{c}\text { Wang et al. } \\
\text { 2013[17] }\end{array}$ & $\begin{array}{c}\text { Discriminant Laplacian } \\
\text { Embedding (DLE) }\end{array}$ & $87.4 \%$ \\
\hline $\begin{array}{c}\text { Razzak and } \\
\text { Hashem 2015 } \\
{[18]}\end{array}$ & $\begin{array}{c}\text { Haar wavelets transform } \\
\text { and Discrete Cosine } \\
\text { Transform (DCT) }\end{array}$ & $\% 92.2$ \\
\hline $\begin{array}{c}\text { Suhaila and. } \\
\text { Dawood 2016 } \\
{[19]}\end{array}$ & Distances and Angles & $91.72 \%$ \\
\hline $\begin{array}{c}\text { The proposed } \\
\text { DESS }\end{array}$ & Distances & $96.39 \%$ \\
\hline
\end{tabular}

The confusion matrix for the best recognition result of the proposed system that given in the table (2).

Table 2: The confusion matrix of the proposed DESS

\begin{tabular}{|c|c|c|c|c|}
\cline { 2 - 5 } \multicolumn{1}{c|}{} & Happy & Angry & Surprise & Sum \\
\hline Happy & 69 & 3 & 2 & 74 \\
\hline Angry & 2 & 72 & 0 & 74 \\
\hline Surprise & 1 & 0 & 73 & 74 \\
\hline Sum & 72 & 75 & 75 & 222 \\
\hline
\end{tabular}

In the table (3) illustrates the percentage of the proposed system the calculated are in the above table (2) by applying equation RR.

Table 3: The percentage of the proposed DESS

\begin{tabular}{|c|c|c|c|}
\cline { 2 - 4 } \multicolumn{1}{c|}{} & Happy & Angry & Surprise \\
\hline Happy & $93 \%$ & $4 \%$ & $3 \%$ \\
\hline Angry & $3 \%$ & $97 \%$ & $0 \%$ \\
\hline Surprise & $1 \%$ & $0 \%$ & $99 \%$ \\
\hline Sum & $32 \%$ & $34 \%$ & $34 \%$ \\
\hline
\end{tabular}

In table (4) shows the percentage of the comparative with the proposed system were found, it's the best of comparability of the previous study [18] of happy, angry, and surprise emotions.

Table 4: Comparison with the proposed DESS

\begin{tabular}{|c|c|c|c|c|c|c|}
\cline { 2 - 7 } \multicolumn{1}{c|}{} & \multicolumn{2}{c|}{ Happy } & \multicolumn{2}{c|}{ Angry } & \multicolumn{2}{c|}{ Surprise } \\
\cline { 2 - 7 } \multicolumn{1}{c|}{} & Propose Model & Comparative Study & Propose Model & ComparativeStudy & Propose Model & ComparativeStudy \\
\hline Happy & $93 \%$ & $92 \%$ & $4 \%$ & $0 \%$ & $3 \%$ & $1 \%$ \\
\hline Angry & $3 \%$ & $0 \%$ & $97 \%$ & $64 \%$ & $0 \%$ & $0 \%$ \\
\hline Surprise & $1 \%$ & $0 \%$ & $0 \%$ & $2 \%$ & $99 \%$ & $94 \%$ \\
\hline
\end{tabular}

In figure (6) illustrates the recognition of the proposed system in the table (3) above.

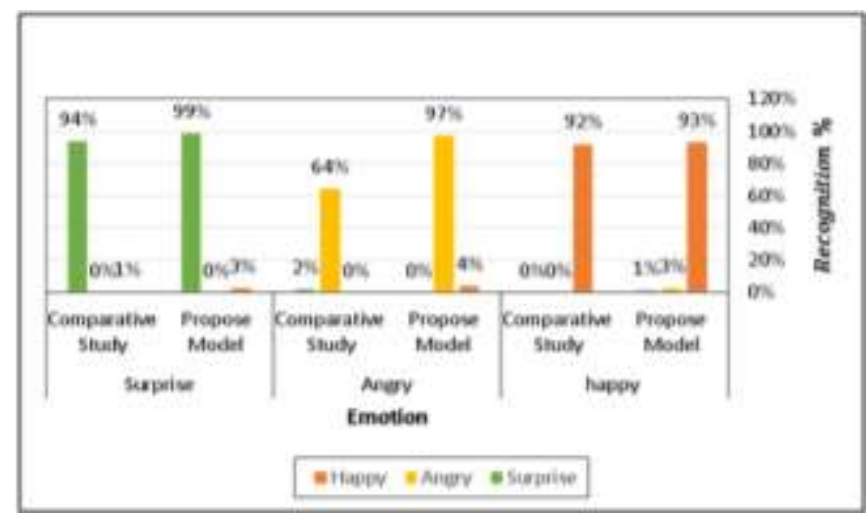

Figure 6: Proposed system comparative other system

In Figure 6 illustration comparative percentage between proposed system and other's:

- In the proposed system the surprise emotion percentage is $99 \%$, while comparative study is $94 \%$.

- The angry emotion percentage in the proposed system is $97 \%$ this percentage is a very high comparative study that is $64 \%$.
- The happy emotion percentage in proposed system is $93 \%$, while comparative study is $92 \%$, this percentage is the best rate of $1 \%$ but will be processed this case in future work.

\section{Conclusions}

The proposed system is constructed to support the education system through detecting the emotion of the students in the classroom and informing the teacher about the emotion type in order to react according to student status. The proposed system directive the teacher to change his/her learning methods to influence the students in the class room. The mood of the students in the classroom measures the abilities of the teacher adjusting what is learned.The images that were used in the implementation, conceptualize the impact of student emotions.

\section{Disclaimer}

The images used in this research belong to the public data base Cohn-Kanade (Ck) which can be downloaded from web site: The Affect Analysis Group at Pittsburgh (http://www.pitt.edu/ emotion/ck-spread.htm). 


\section{International Journal of Science and Research (IJSR) \\ ISSN (Online): 2319-7064}

Index Copernicus Value (2016): 79.57 | Impact Factor (2015): 6.391

\section{References}

[1] Z. Zeng, M. Pantic, G. Roisman \& T. Huang. A survey of affect recognition methods: Audio, visual, and spontaneous expressions. IEEE Transactions on Pattern Analysis and Machine Intelligence, 31, 1 (2009), 39-58.

[2] Murty G, Kiran J, Kumar V. Facial expression recognition based on features derived from the distinct LBP and GLCM. Image, Graphics and Signal Processing. 2014;2:68-77.

[3] Guangda HG, Du SC. Feature points extraction from faces. Image and Vision Computing. 2003;154-158.

[4] Iatraki G. Emotional facial expression recognition $\&$ classification. Department of Media and Knowledge Engineering. Delft University of Technology; 2009.

[5] Youssif AA, Asker WA. Automatic facial expression recognition system based on geometric and appearance features. Computer and Information Science. 2011; 4(2):115-124.

[6] Shan C, Gong S, McOwan PW. Facial expression recognition based on local binary patterns: A comprehensive study. Image and Vision Computing. 2009; 27(6):803-816.

[7] S. Kranthi, K. Pranathi, and A. Srisaila, "Automatic Number Plate Recognition", International Journal of Advancements in Technology, Vol. 2, No. 3, PP. 408-422, July, 2011

[8] R. Gonzalez, and R. Woods, "Digital Image Processing", Second Edition, Prentice Hall, 2002.

[9] Rupinderpal, K.; and Rajneet, K.; "Survey of Denoising Methods Using Filters and Fast Wavelet Transform"; International Journal of Advanced Research in Computer Science and Software Engineering (IJARCSSE), Vol. 3, Iss. 2, PP. 133136, 2013.

[10] E. Nadernejad, S. Sharifzadeh and H. Hassanpour, "Edge Detection Techniques Evaluations and Comparisons", Applied Mathematical Sciences, Vol. 2, No. 31, PP. 1507-1520, 2008.

[11] J. Shi,N. Ray ,H. Zhang, "Shape based local thresholding for binarization of document images", International Journal of Selsvevier, Issue 1, Vol. 33,Pp. 24-32, January 2012.

[12] Langote, V. B.; and Chaudhari, D. S.; "Segmentation Techniques for Image Analysis"; International Journal of Advanced Engineering Research and Studies (IJAERS), Vol. 1, Issue 11, Pp. 252-255, 2012

[13] Viswanathan DM, Idicula SM. Automatic facial expression analysis: A survey. International Journal of Image Processing (IJIP). 2015;9(1):32-40.

[14] Verma V, Sharma LK. A comprehensive survey on human facial expression detection. International Journal of Image Processing (IJIP). 2013;7(2):171182.

[15] Sharma, P.; Singh, G. and Kaur, A.; "Different Techniques of Edge Detection in Digital Image Processing"; International Journal of Engineering Research and Applications (IJERA), Vol. 3, Issue 3, Pp. 458-461, May-June 2013.
[16] Cetingul, H. E.; Yemez, Y.; Erzin, E.; and Tekalp, A. M.; "Discriminative Analysis of Lip Motion Features for Speaker Identification and SpeechReading"; IEEE Transactions On Image Processing, Vol. 15, No. 10, Pp. 2879-2891, October 2006.

[17] Wang H, Huang H, Makedon F. Emotion detection via discriminant Laplacian embedding. Springer. 2013;440- 452.

[18] Razzak AA, Hashem AR. Facial expression recognition using hybrid transform. International Journal of Computer Applications. 2015; 119(15): 12- 18.

[19] S. N. Mohammed, L. E. George and H. A. Dawood, "The Effect of Classification Methods on Facial emotion Recognition Accuracy", accepted of British Journal of Applied Science \& Technology, Vol.14, Iss. 4, PP.1-11, 2016

Volume 6 Issue 12, December 2017 www.ijsr.net 